\title{
CODAZZI TENSORS WITH TWO EIGENVALUE FUNCTIONS
}

\author{
GABE MERTON
}

(Communicated by Lei $\mathrm{Ni}$ )

\begin{abstract}
This paper addresses a gap in the classification of Codazzi tensors with exactly two eigenfunctions on a Riemannian manifold of dimension three or higher. Derdzinski proved that if the trace of such a tensor is constant and the dimension of one of the eigenspaces is $n-1$, then the metric is a warped product where the base is an open interval, a conclusion we will show to be true under a milder trace condition. Furthermore, we construct examples of Codazzi tensors having two eigenvalue functions, one of which has eigenspace dimension $n-1$, where the metric is not a warped product with interval base, refuting a claim by A. L. Besse that the warped product conclusion holds without any restriction on the trace.
\end{abstract}

\section{INTRODUCTION}

A symmetric $(0,2)$ tensor $A$ is Codazzi if it satisfies the symmetry property

$$
\left(\nabla_{X} A\right)(Y, Z)=\left(\nabla_{Y} A\right)(X, Z)
$$

for any vector fields $X, Y$ and $Z$. Alternatively, a $(1,1)$ tensor $A$ is Codazzi if it is self-adoint and

$$
\left(\nabla_{X} A\right) Y=\left(\nabla_{Y} A\right) X
$$

Throughout the paper, $V_{\lambda}$ denotes the eigendistribution corresponding to the eigenvalue function $\lambda$ of the tensor $A$. That is, we say a vector field $Y$ is in $V_{\lambda}$ if $A Y=\lambda Y$. While it's conceptually more appropriate to view Codazzi tensors as $(1,1)$, computations are often easier when the tensor is viewed as $(0,2)$.

There are many well-known examples of Codazzi tensors: any constant scalar multiple of the metric and, more generally, any parallel self-adjoint $(1,1)$ tensor. One may also ask what it means if certain well-known self-adjoint $(1,1)$ tensors are Codazzi. For example, the second fundamental form of a hypersurface embedded in a space of constant sectional curvature is Codazzi. When the larger space is $\mathbb{R}^{3}$, this is the content of the famous Codazzi-Mainardi equation. It's a standard exercise in Riemannian geometry to show that Ric is Codazzi if and only if the divergence of the full curvature tensor vanishes; i.e. the curvature is harmonic. This is the case, for example, on Einstein manifolds.

Received by the editors November 29, 2011.

2010 Mathematics Subject Classification. Primary 53A45, 53B20.

(C) 2013 American Mathematical Society Reverts to public domain 28 years from publication 
There are other more subtle relationships between the behavior of Codazzi tensors and the topology and geometry of the manifold. Berger-Ebin proved in [1] that a constant trace Codazzi tensor on a compact manifold with non-negative sectional curvature must be parallel. As a nice low-dimension result, Bourguignon showed in [3] that a compact orientable four-manifold admitting a non-trivial Codazzi tensor with constant trace must have signature zero. Derdzinski-Shen proved in 6 that if a Codazzi tensor on $M^{n}$ has $n$ distinct eigenvalues at all points of $M$, then all the Pontryagin classes of $M$ are zero.

Another result relating the geometry of the manifold to a Codazzi tensor's spectrum is the departure point of this paper.

Theorem 1.1 (Derdzinski). Suppose $A$ is a Codazzi tensor on $M^{n}, n \geq 3$, having exactly two distinct eigenvalue functions $\lambda, \mu$ in a neighborhood of $p$ with $\operatorname{dim} V_{\mu} \leq$ $\operatorname{dim} V_{\lambda}$. Then, there exists a neighborhood of $p$ such that

i. $M$ is a Riemannian product if and only if $\operatorname{dim} V_{\mu} \geq 2$ or $A$ is parallel in a neighborhood of $p$.

ii. $M$ is a warped product with interval base and non-trivial warping function if and only if $\operatorname{dim} V_{\mu}=1, A$ has constant trace, and $A$ is not parallel. In this case, $M=I \times_{F} N$, where $N$ and $I$ are the integral submanifolds of $V_{\lambda}$ and $V_{\mu}$, respectively.

It's not immediately obvious that such integral manifolds exists. However, a well-known property of Codazzi tensors that we'll review in Section 2 is the fact that their eigendistributions are integrable.

Theorem 1.1 was first proved by Derdzinski in [5] and is reported in Besse's Einstein Manifolds [2]. Besse precedes the proof with the statement "A similar argument works without the hypothesis [that trace $A$ is constant]." This statement can be interpreted in two ways. First some terminology. If $A$ is a Codazzi tensor with exactly two distinct eigenfunctions $\mu$ and $\lambda$ and if $M=M_{1} \times_{F} M_{2}$ is a warped product, we will say that the warped product and eigenspace structures are consistent if $M_{1}$ and $M_{2}$ are integral submanifolds of the eigendistributions $V_{\mu}$ and $V_{\lambda}$. In Theorem 1.1, the structures are consistent. The remark by Besse could be saying that without the constant trace assumption,

i. $M$ is either a product or a warped product with interval base or

ii. $M$ is either a product or a warped product with interval base, and the warping variable is the coordinate of the interval.

In Section 4, we prove the following propositions showing that neither statement is true.

Proposition 1. There exists a compact Riemannian manifold $(M, g)$ and a Codazzi tensor $A$ with exactly two distinct eigenfunctions $\mu$ and $\lambda$ with $\operatorname{dim} V_{\mu}=1$ such that $M$ is neither a product nor a warped product.

Proposition 2. There exists a Riemannian manifold $(M, g)$ and a Codazzi tensor $A$ with exactly two distinct eigenfunctions $\mu$ and $\lambda$ with $\operatorname{dim} V_{\mu}=1$ such that $M$ is a warped product with interval base, but the warped product structure is inconsistent with the eigenspace structure.

However, the conclusion of Theorem 1.1 does hold under conditions weaker than the trace being constant. In Section 3 we prove the following theorem. 
Theorem 1.2. Let $A$ be a Codazzi tensor on a manifold $M^{n}, n \geq 3$. Suppose there exists a neighborhood of a point $p$ where $A$ has two distinct eigenfunctions $\mu$ and $\lambda, \operatorname{dim} V_{\mu}=1$, and $\lambda$ is not constant. Assume that any one of the following conditions holds:

(1) $D_{Y}(\operatorname{tr}(A))=0$ for all $Y \in V_{\lambda}$.

(2) $D_{Y} \mu=0$ for all $Y \in V_{\lambda}$.

(3) The integral curves of $V_{\mu}$ are geodesics.

(4) If for every unit vector $X \in V_{\mu}$ there exists a function $f \in C^{\infty}(M)$ such that locally $\nabla f=X$.

Then the metric is a non-trivial warped product with interval base consistent with the eigenspace structure of $A$.

In the theorem, the condition that $\lambda$ be non-constant replaces the condition that $A$ be non-parallel in the constant trace case and guarantees that the warping function is non-trivial.

\section{BACKGROUND}

In this section we assemble the tools needed to prove the proposition and the theorem. All the results in this section can be found in [5] or [2] and seem to have appeared first in [7], though the assumptions are slightly different 1 Lemma 2.1 gives a formula for the image of $\nabla_{Y} X$ under the Codazzi tensor $A$ given that $X$ and $Y$ are eigenvectors of the same eigenfunction.

Lemma 2.1. Suppose $A$ is a Codazzi tensor and that $X$ and $Y$ are two sections in $V_{\lambda}$. Then

$$
A \nabla_{Y} X=\lambda \nabla_{Y} X+\left(D_{Y} \lambda\right) X-g(X, Y) \nabla \lambda .
$$

The next lemma shows that as long as the dimension of the eigendistribution $V_{\lambda}$ is at least two, the behavior of the eigenfunction $\lambda$ is severely restricted in the sense that its directional derivative is zero along any direction belonging to $V_{\lambda}$. When we discuss the particular case of interest where there are only two eigenfunctions $\mu$ and $\lambda$ with $\operatorname{dim} V_{\mu}=1$ and $\operatorname{dim} V_{\lambda}=n-1$, the lemma implies that there's only one linearly independent direction in which $\lambda$ can vary.

Lemma 2.2. If $A$ is a Codazzi tensor and $V_{\lambda}$ is the eigendistribution of the eigenfunction $\lambda, \operatorname{dim} V_{\lambda} \geq 2$, then $D_{Y} \lambda=0$ for all $Y \in V_{\lambda}$.

Lemmas 2.1 and 2.2 are used to prove that the eigendistributions of Codazzi tensors are integrable.

Theorem 2.3. The eigendistributions of a Codazzi tensor A are integrable.

Note that by combining Theorem 2.3 and Lemma 2.2. one can say that if dim $V_{\lambda} \geq 2$, then $\lambda$ is constant along the leaves of $V_{\lambda}$.

The final technical lemma gives a formula for the directional derivative of an eigenfunction $\lambda$ when the direction, $Y$, belongs to a different eigendistribution.

Lemma 2.4. If $A$ is a Codazzi tensor with $Y \in V_{\lambda}$ and $X, Z \in V_{\mu}$, then

$$
D_{Y} \mu \cdot g(X, Z)=(\lambda-\mu) g\left(\nabla_{X} Y, Z\right) .
$$

\footnotetext{
${ }^{1}$ The author wishes to thank Andrzej Derdzinski for bringing this reference to his attention.
} 


\section{Removing the CONStant trace ASSUmption}

We now turn to the proof of Theorem 1.2, The proof consists of two steps. In Proposition 3, four conditions are shown to be equivalent. We then use the proposition to show the existence of a warped product structure.

It's straightforward to see that the trace of $A$, where $A$ possesses exactly two eigenfunctions $\mu$ and $\lambda$ with $\operatorname{dim} V_{\mu}=1$ and $\operatorname{dim} V_{\lambda}=n-1$, is

$$
\text { trace } A=\mu+(n-1) \lambda \text {. }
$$

If the trace is constant and $Y \in V_{\lambda}$, then by Lemma 2.2, $Y \mu=0$. That is, the constant trace assumption implies $Y \mu=0$ for all $Y \in V_{\lambda}$. Theorem 1.2 shows this conclusion, that $Y \mu=0$ is sufficient to obtain a warped product structure. Alternatively, using the first characterization given in Proposition [3, it's sufficient that the trace be constant in all directions except for one.

Proposition 3. Let $A$ be a Codazzi tensor on a manifold $M^{n}, n \geq 3$, with two distinct eigenfunctions $\mu$ and $\lambda$ on an open domain $W$. Assume dim $V_{\mu}=1$. The following are equivalent:

(1) $D_{Y}(\operatorname{tr}(A))=0$ for all $Y \in V_{\lambda}$.

(2) $D_{Y} \mu=0$ for all $Y \in V_{\lambda}$.

(3) The integral curves of $V_{\mu}$ are geodesics.

(4) If $X \in V_{\mu},|X|=1$, then there exists a function $f \in C^{\infty}(M)$ such that locally $\nabla f=X$.

Proof. Throughout the proof assume $X$ is a unit vector in $V_{\mu}$ and $Y, Z \in V_{\lambda}$.

$(1) \Leftrightarrow(2)$. This follows from the equation

$$
\operatorname{tr}(A)=\mu+(n-1) \lambda
$$

and the fact that $D_{Y} \lambda=0$ for $Y \in V_{\lambda}$.

$(2) \Leftrightarrow(3) \cdot g\left(\nabla_{X} X, X\right)=0$ since $|X|=1$. By Lemma 2.4.

$$
D_{Y} \mu=(\mu-\lambda) g\left(\nabla_{X} X, Y\right) \text {. }
$$

Since $\mu \neq \lambda, \nabla_{X} X=0$ if and only if $Y \mu=0$.

$(4) \Leftrightarrow(2)$. To show this implication, recall that a vector field $X$ is gradient if and only if $\nabla X$ is symmetric. We have

$$
\begin{aligned}
\left(\nabla_{Y} A\right)(X, Z) & =-A\left(\nabla_{Y} X, Z\right)-A\left(X, \nabla_{Y} Z\right) \\
& =-\lambda g\left(\nabla_{Y} X, Z\right)-\mu g\left(X, \nabla_{Y} Z\right) \\
& =(\mu-\lambda) g\left(\nabla_{Y} X, Z\right) .
\end{aligned}
$$

Similarly, $\left(\nabla_{Z} A\right)(X, Y)=(\mu-\lambda) g\left(\nabla_{Z} X, Y\right)$. By the Codazzi condition,

$$
g\left(\nabla_{Z} X, Y\right)=g\left(\nabla_{Y} X, Z\right) \text {. }
$$

Thus, as an $(0,2)$ tensor, $\nabla X$ is symmetric on $V_{\lambda} \times V_{\lambda}$ regardless of conditions (1)-(3). We also have

$$
\begin{aligned}
& g\left(\nabla_{X} X, Y\right)=(\mu-\lambda)^{-1} D_{Y} \mu, \\
& g\left(\nabla_{Y} X, X\right)=\frac{1}{2} Y g(X, X)=0 .
\end{aligned}
$$

This shows $\nabla X$ is symmetric if and only if $Y \mu=0$.

We now have everything we need to prove the main theorem. 
Proof of Theorem 1.2. In general, the eigenbundles of a Codazzi tensor are integrable and orthogonal, so there exists a chart $\left\{U, r, y_{1}, \ldots, y_{n-1}\right\}$ such that $\partial_{r} \in V_{\mu}$ and $\partial_{i}=\partial_{y_{i}} \in V_{\lambda}$. Let $X \in V_{\mu}$ be a unit vector field. By the fourth criterion in Proposition $3, X=\nabla t$ for some local submersion $t: U \rightarrow \mathbb{R}$. Now if $Y \in V_{\lambda}$, then $Y t=g(X, Y)=0$, meaning there exist coordinates $\left\{t, x_{1}, \ldots, x_{n-1}\right\}$ such that $\partial_{t} \in V_{\mu}, \partial_{x_{i}} \in V_{\lambda}$ and $g\left(\partial_{t}, \partial_{t}\right)=1$. Moreover, $A\left(\partial_{t}, \partial_{j}\right)=g\left(\partial_{t}, \partial_{j}\right)=0$ and $A\left(\partial_{i}, \partial_{j}\right)=\lambda(t) g_{i j}$.

The next step is to prove that $\partial_{t} g_{i j}=f(t) g_{i j}$. Lemma 2.4 implies

$$
\begin{aligned}
\partial_{t} g\left(\partial_{i}, \partial_{j}\right) & =-2 g\left(\nabla_{\partial_{i}} \partial_{j}, \partial_{t}\right) \\
& =2(\mu-\lambda)^{-1}\left(\partial_{t} \lambda\right) g_{i j}=2 \eta g_{i j},
\end{aligned}
$$

where $\eta=(\mu-\lambda)^{-1}\left(\partial_{t} \lambda\right)$. We can write

$$
\eta g_{i j}=-g\left(\nabla_{\partial_{i}} \partial_{j}, \partial_{t}\right)=\operatorname{Hesst}\left(\partial_{i}, \partial_{j}\right) .
$$

Now show that $\eta$ depends only on $t$ :

$$
\begin{aligned}
\partial_{i} \eta & =-(\mu-\lambda)^{-2} \cdot\left(\partial_{i} \mu-\partial_{i} \lambda\right)\left(\partial_{t} \lambda\right)+(\mu-\lambda)^{-1}\left(\partial_{i} \partial_{t} \lambda\right) \\
& =(\mu-\lambda)^{-1}\left(\partial_{i} \partial_{t} \lambda\right) \\
& =(\mu-\lambda)^{-1}\left(\partial_{t} \partial_{i} \lambda\right)=0 .
\end{aligned}
$$

Since $\partial_{t} g_{i j}=\eta(t) g_{i j}$, integrate $\eta$ to obtain a function $q(t)$ such that $\partial_{t}\left(e^{-q} g_{i j}\right)=$ 0 . This means $g_{i j}=e^{q(t)} h_{i j}$ for some $h_{i j}$. This shows $M$ is a warped product. The warping function is trivial if and only if $\eta=0$, which happens if and only if $\lambda$ is constant.

\section{Counterexamples}

This section presents a class of Codazzi tensors on open sets of $\mathbb{R}^{3}$ that provide the source of counterexamples for Propositions 1 and 2.

Let $\lambda>0$ be a constant and $\mu(t, x, y)$ be a $C^{\infty}$ function on an open set $V \subset \mathbb{R}^{3}$ and such that there exists a connected open set $U \subset V$ where $\mu \neq \lambda$. Define a metric and tensor on $U$ by

$$
\begin{aligned}
g & =(\lambda-\mu(t, x, y))^{-2} d t^{2}+\lambda d x^{2}+\lambda d y^{2}, \\
A\left(\partial_{t}\right) & =\mu(t, x, y) \partial_{t}, \\
A\left(\partial_{x}\right) & =\lambda \partial_{x}, \\
A\left(\partial_{y}\right) & =\lambda \partial_{y} .
\end{aligned}
$$

As a step toward proving that $A$ is indeed Codazzi, calculate the Christoffel symbols. Throughout this section, the subscripts $i, j$ and $k$ shall refer to the variables $x$ and $y$. For example, $\partial_{i}$ could mean either $\partial_{x}$ or $\partial_{y}$ but not $\partial_{t}$.

Lemma 4.1. The non-trivial Christoffel symbols of this metric are

$$
\begin{aligned}
& \Gamma_{t t}^{t}=(\lambda-\mu)^{-1}\left(\partial_{t} \mu\right), \\
& \Gamma_{t t}^{i}=-\frac{1}{\lambda}(\lambda-\mu)^{-3}\left(\partial_{i} \mu\right), \\
& \Gamma_{i t}^{t}=(\lambda-\mu)^{-1}\left(\partial_{i} \mu\right) .
\end{aligned}
$$

Proposition 4. The tensor $A$ defined above is Codazzi. 
Proof. It suffices to prove $\left(\nabla_{X} A\right)(Y, Z)=\left(\nabla_{Y} A\right)(X, Z)$, where $X, Y$ and $Z$ are all coordinate vectors. Again, let $\partial_{i}, \partial_{j}$ and $\partial_{k}$ indicate partial derivatives with respect to $x$ or $y$. Straightforward calculations show that

i. $\left(\nabla_{\partial_{t}} A\right)\left(\partial_{i}, \partial_{j}\right)=\left(\nabla_{\partial_{i}} A\right)\left(\partial_{t}, \partial_{j}\right)=0$.

ii. $\left(\nabla_{\partial_{i}} A\right)\left(\partial_{t}, \partial_{t}\right)=\left(\nabla_{\partial_{t}} A\right)\left(\partial_{i}, \partial_{t}\right)=\left(\partial_{i} \mu\right) g_{t t}$.

iii. $\left(\nabla_{\partial_{i}} A\right)\left(\partial_{j}, \partial_{k}\right)=0$.

Our strategy for proving Propositions 1 and 2 will be to judiciously select $\mu$ and $\lambda$ along with the following well-known characterization of warped products with interval bases, first proved by Brinkmann in [4.

Lemma 4.2. The following are equivalent:

A. There exists a neighborhood $V$ of $p$ and a function $f$ such that Hess $f=a \cdot g$ for some function $a$ and $\nabla f(p) \neq 0$.

B. There exists a neighborhood $V$ of $p$ such that $V$ is a warped product space $V=I \times{ }_{w} F$ with 1-dimensional base $I$.

Thus, to show that a metric is not an interval warped product, it suffices to show that if $f$ and $a$ satisfy Hess $f=a \cdot g$, then $\nabla f=0$ in a neighborhood; i.e. $f$ is locally constant.

Lemma 4.3. The components of Hess $f$ for the metric given above are

$$
\begin{aligned}
& \text { Hess } f\left(\partial_{x}, \partial_{x}\right)=f_{x x}, \\
& \text { Hess } f\left(\partial_{y}, \partial_{y}\right)=f_{y y}, \\
& \text { Hess } f\left(\partial_{x}, \partial_{y}\right)=f_{x y}, \\
& \text { Hess } f\left(\partial_{t}, \partial_{t}\right)=f_{t t}-(\lambda-\mu)^{-1} \mu_{t} f_{t}+\frac{1}{\lambda} \cdot(\lambda-\mu)^{-3}\left(f_{x} \mu_{x}+f_{y} \mu_{y}\right), \\
& \text { Hess } f\left(\partial_{t}, \partial_{x}\right)=f_{t x}-(\lambda-\mu)^{-1} \mu_{x} f_{t}, \\
& \text { Hess } f\left(\partial_{t}, \partial_{y}\right)=f_{t y}-(\lambda-\mu)^{-1} \mu_{y} f_{t} .
\end{aligned}
$$

Proof. Use the formula, Hess $f\left(\partial_{i}, \partial_{j}\right)=\partial_{i} \partial_{j} f-\Gamma_{i j}^{k} \partial_{k} f$, and the Christoffel symbols calculated above.

From here on we will study the particular case where $\lambda=1$ and $\mu(t, x, y)=$ $\mu(x, y)$. Then by Lemma 4.2 , if we are given a particular $\mu(x, y)$ we should look for functions $f(t, x, y)$ and $a(t, x, y)$ that solve the system below:

$$
\begin{aligned}
f_{x x} & =a, \\
f_{y y} & =a, \\
f_{x y} & =0, \\
f_{t y}(1-\mu) & =\mu_{y} f_{t}, \\
f_{t x}(1-\mu) & =\mu_{x} f_{t}, \\
f_{t t}+(1-\mu)^{-3}\left(f_{x} \mu_{x}+f_{y} \mu_{y}\right) & =a(1-\mu)^{-2} .
\end{aligned}
$$

Proposition 5. If for a given $\mu(x, y)$ there exists $f$ satisfying the system of PDE's given above, with $\nabla f(0) \neq 0$, then $\mu$ must be in one of the following forms:

1. $\mu(x, y)=1+\frac{c_{1}}{1-c_{3} x-c_{4} y-c_{2}\left(x^{2}+y^{2}\right)}$, 
2. $\mu(x, y)=\frac{a x+G\left(\frac{c+a y}{a(b+a x)}\right)}{a x+b}$,

3. $\mu(x, y)=\frac{a y+G\left(\frac{x}{c+a y}\right)}{c+a y}$,

4. $\mu(x, y)=G\left(\frac{b y-c x}{b}\right)$

5. $\mu(x, y)=\mu(x)$,

6. $\mu(x, y)=\mu(y)$.

Proof. The first three Hessian equations,

$$
\begin{aligned}
& f_{x x}=a g\left(\partial_{x}, \partial_{x}\right)=a, \\
& f_{x y}=a g\left(\partial_{x}, \partial_{y}\right)=0, \\
& f_{y y}=a,
\end{aligned}
$$

collectively imply $a(t, x, y)=a(t)$ and

$$
f(t, x, y)=\frac{a(t)}{2}\left(x^{2}+y^{2}\right)+b(t) x+c(t) y+k(t) .
$$

If we write $h(t, x, y)=f_{t}(t, x, y)$, then we can rewrite and solve the fourth Hessian equation as a first order differential equation:

$$
\begin{aligned}
h_{y}(1-\mu) & =\mu_{y} h, \\
h_{y} & =\mu_{y} h+\mu h_{y}=\frac{\partial}{\partial y}(\mu h) \\
\Rightarrow f_{t}(t, x, y) & =h(t, x, y)=C_{1}(t, x) /(\mu(x, y)-1) .
\end{aligned}
$$

An analogous argument works for the fifth equation, so

$$
f_{t}(t, x, y)=\frac{C_{1}(t, x)}{\mu(x, y)-1}=\frac{C_{2}(t, y)}{\mu(x, y)-1} \Rightarrow f_{t}(t, x, y)=\frac{C_{1}(t)}{\mu(x, y)-1} .
$$

We can solve for $C_{1}(t)$ by using the fact that $f_{t}(t, x, y)$ is a polynomial and evaluating at $(t, 0,0)$ :

$$
\begin{aligned}
\frac{C_{1}(t)}{\mu(x, y)-1}= & \frac{a^{\prime}(t)}{2}\left(x^{2}+y^{2}\right)+b^{\prime}(t) x+c^{\prime}(t) y+k^{\prime}(t) \\
& \Rightarrow \frac{C_{1}(t)}{\mu(0,0)-1}=k^{\prime}(t) \\
& \Rightarrow C_{1}(t)=(\mu(0,0)-1) k^{\prime}(t) .
\end{aligned}
$$

Letting $c_{1}=\mu(0,0)-1$, we have

$$
f_{t}(t, x, y)=\frac{c_{1} k^{\prime}(t)}{\mu-1} \Rightarrow f(t, x, y)=\frac{c_{1} k(t)}{\mu-1}+K(x, y) .
$$

Equating to the polynomial expression for $f$ gives us

$$
\begin{aligned}
\frac{c_{1} k(t)}{\mu-1}+K(x, y) & =\frac{a(t)}{2}\left(x^{2}+y^{2}\right)+b(t) x+c(t) y+k(t) \\
\Rightarrow K(x, y) & =\frac{a(t)}{2}\left(x^{2}+y^{2}\right)+b(t) x+c(t) y+H(x, y) k(t),
\end{aligned}
$$


where $H(x, y)=1-\frac{c_{1}}{\mu-1}$. Take a $t$ derivative of each side to get a linear equation of the functions $x^{2}+y^{2}, x, y$ and $H(x, y)$ :

$$
\frac{a^{\prime}(t)}{2}\left(x^{2}+y^{2}\right)+b^{\prime}(t) x+c^{\prime}(t) y+H(x, y) k^{\prime}(t)=0 .
$$

This equation implies either $a^{\prime}(t)=b^{\prime}(t)=c^{\prime}(t)=k^{\prime}(t)=0$ or

$$
H(x, y)=c_{2}\left(x^{2}+y^{2}\right)+c_{3} x+c_{4} y .
$$

We can now solve for $\mu$, since

$$
\begin{aligned}
H & =1-\frac{c_{1}}{\mu-1} \\
\Rightarrow \mu(x, y) & =1+\frac{c_{1}}{1-H} \\
& =1+\frac{c_{1}}{1-c_{3} x-c_{4} y-c_{2}\left(x^{2}+y^{2}\right)} .
\end{aligned}
$$

A continued analysis would investigate the implications of the final Hessian equation. However, the equation for $\mu$ just derived will be sufficient for our purposes.

We now investigate solutions to the system when $a, b, c$ and $k$ are all constant. This simplifies the system considerably. The third and fourth equations automatically hold, and the final equation simplifies to

$$
(a x+b) \mu_{x}+(a y+c) \mu_{y}=a(1-\mu) .
$$

This PDE is straightforward to solve by using the Method of Characteristics:

$$
\begin{aligned}
& \mu(x, y)=\frac{a x+G\left(\frac{c+a y}{a(b+a x)}\right)}{a x+b} \text { if } a \neq 0 \text { and } b \neq 0, \\
& \mu(x, y)=\frac{a y+G\left(\frac{x}{c+a y}\right)}{c+a y} \text { if } a \neq 0 \text { and } b=0, \\
& \mu(x, y)=G\left(\frac{b y-c x}{b}\right) \text { if } a=0 \text { and } b \neq 0, \\
& \mu(x, y)=\mu(x) \text { if } a=0, b=0 \text { and } c \neq 0, \\
& \mu(x, y)=\mu(y) \text { if } a=0, b \neq 0 \text { and } c=0 .
\end{aligned}
$$

By selecting any $\mu$ not in one of the above forms, we can generate an example of a Codazzi tensor on a compact manifold where the metric is not a warped product at least at one point.

Corollary 4.4. Let $\mu(x, y)=\frac{1}{2} \sin x \cos y$. Then the metric $g$ and Codazzi tensor $A$ defined above are periodic and pass to a metric $\bar{g}$, and Codazzi tensor $\bar{A}$ on $S^{1} \times S^{1} \times S^{1} \cdot \bar{g}$ is not a warped product on a neighborhood of the point $[(0,0,0)]$.

This result follows from the fact that $\mu(x, y)$ is clearly not in any of the functional forms listed in the proposition.

For the proof of Proposition 2 we use the same template but with $\mu=1+\left(y / x^{2}\right)$. As before, $\lambda=1$. 
Proof of Proposition 2, Let $M=\left\{(t, x, y) \in \mathbf{R}^{3}: y \neq 0\right\}$. Define a metric and tensor on $M$ by

$$
\begin{aligned}
& g=\frac{x^{4}}{y^{2}} d t^{2}+d x^{2}+d y^{2}, \\
& A \partial_{t}=\left(1+\frac{y}{x^{2}}\right) \partial_{t}, \\
& A \partial_{x}=\partial_{x}, \\
& A \partial_{y}=\partial_{y} .
\end{aligned}
$$

It's clear that the metric cannot be written in the form

$$
g=d t^{2}+F(t)\left(d x^{2}+d y^{2}\right),
$$

so either the metric is a warped product with inconsistent warping and eigenspace structures or the metric is not warped at all. If we let $x=r \cos \theta$ and $y=r \sin \theta$, then

$$
g=d r^{2}+r^{2}\left(\frac{\cos ^{4} \theta}{\sin ^{2} \theta} d t^{2}+d \theta^{2}\right) .
$$

This shows the metric is warped in the $r$ direction.

\section{REFERENCES}

[1] M. Berger and D. Ebin, Some decompositions of the space of symmetric tensors on a Riemannian manifold, J. Differential Geometry 3 (1969) 397-392. MR.0266084 (42:993)

[2] A.L. Besse. Einstein Manifolds, Springer, 1987. MR867684 (88f:53087)

[3] J. Bourguignon, Les variétés de dimension 4 à signature non nulle dont la courbure est harmoniq sont d'Einstein, Invent. Math. 63, no. 2 (1981) 263-286. MR610539 (82g:53051)

[4] H.W. Brinkmann, Einstein spaces which are mapped conformally on each other, Math. Ann. 94 (1925) 119-145. MR 1512246

[5] A. Derdzinski, Some remarks on the local structure of Codazzi tensors, in Global Differential Geometry and Global Analysis, Lecture Notes in Math., no. 838, Springer (1981) 251-255. MR0636259(82i:53001)

[6] A. Derdzinski and C.L. Shen, Codazzi tensor fields, curvature and Pontryagin forms, Proc. London Math. Soc. 47, no. 3 (1983) 15-26. MR698925 (84h:53048)

[7] H. Reckziegel, Krümmungsflächen von isometrischen Immersionen in Räume konstanter Krümmung, Math. Ann. 223 (1976), no. 2, 169-181. MR0425846 (54:13796)

Department of Mathematics, University of Calfornia, los Angeles, Box 951555,

Los Angeles, California 90095-1555

E-mail address: gmertonus@yahoo.com 